\title{
MAGNETIC FIELDS IN X-RAY BINARY SYSTEMS
}

\author{
JAMES C. KEMP* \\ Institute for Astronomy, University of Hawaii, Honolulu, H.I. 96822, U.S.A.
}

\begin{abstract}
The case for variable magnetic fields, in the range 1-15 kG, in three of the $\mathrm{X}$-ray binary candidate stars, is discussed. We refer to electronic Zeeman polarimetry by Kemp and Wolstencroft (1973a and b) and by Angel et al. (1973). Our detections in $\mathrm{H} \beta$ showed: (1) definite magnetic fields, roughly constant over periods of $2 \mathrm{~h}$, in $\theta^{2}$ Orionis and HD 77581; and (2) complex time behavior in HD 153919, with field reversals over periods of $\sim 10 \mathrm{~min}$, but with usually small values ( $\lesssim 2 \mathrm{kG}$ ) as averaged over times of 1-2 h. (Photographic Zeeman spectroscopy is therefore unlikely to show detectable fields, i.e. $>2 \mathrm{kG}$, in the latter star.) The essentially null results of Angel et al. (1973) in H $\alpha$, in HD 77581 and HD 153919, indicate that the emission is non-magnetic; only the underlying absorption (which is far weaker than the emission in $\mathrm{H} \alpha$ ) shows Zeeman effects. A plot of the Zeeman measure for HD 77581, against the 8.95-day period, is shown: our points are suggestive of periodicity; and the points of Angel et al. (1973) which unfortunately have larger relative error bars, are consistent with the indicated variation and with the general scale of our results.
\end{abstract}

\section{References}

Angel, J. R. P., McGraw, J. T., and Stockman, H. S. Jr.: 1973, Astrophys. J. Letters 184, L79.

Kemp, J. C. and Wolstencroft, R. D.: 1973a, Astrophys. J. Letters 182, L43.

Kemp, J. C. and Wolstencroft, R. D. : 1973b, Astrophys. J. Letters 185, L21.

\section{DISCUSSION}

Buscombe: I would appeal to you to tackle some of supergiant A stars I was talking about yesterday. because I think you would see some activity of this kind in the $\mathrm{H} \alpha$.

Kemp: We have.

Buscombe: What do you get?

Kemp: 25 Orionis. We see enormous effects, we haven't published them yet. That is a Be emission star; we have several more. $\delta$ Cas is another one. It's a rapid rotator. They're non-periodic, that's one reason we haven't said anything about them.

Buscombe: The lines are so broadened by turbulence that you'd have no hope with the conventional magnetic analyser.

Kemp: What we see is all kinds of fantastic time variations, linear polarization effects particularly in the Balmer lines.

Buscombe: You have problems with the depolarization in the interstellar medium because these are distant supergiants.

Kemp: Yes, in all these cases there is a large interstellar polarization which one has to deal with.

* Also Department of Physics. University of Oregon, Eugene, Oregon 97403. 
Tayler: Do you have any estimate at all of the strength of the field you're looking at?

Kemp: Yes, I didn't say anything about this, and we were overly enthusiastic in assigning field strengths to these results in the first paper. It's very complicated because of the spectrum. However, these fields roughly correspond to $1-15 \mathrm{kG}$. It's very sensitive to this whole question of the emission and absorption components. You can either have a region where you see where the magnetic field is falling off in some sense and there is emission and you get a negative contribution. If you assume an equivalent width of about $1 \AA$ in $\mathrm{H} \beta$ in emission, these correspond to fields around a couple of $\mathrm{kG}$. 